Asian J. Med. Biol. Res. 2021, 7 (1), 76-81; doi: 10.3329/ajmbr.v7i1.53312

\author{
Asian Journal of \\ Medical and Biological Research \\ ISSN 2411-4472 (Print) 2412-5571 (Online) \\ www.ebupress.com/journal/ajmbr
}

\title{
Article \\ The correlation of bone mineral density, body mass index and age
}

\author{
S. Sultana ${ }^{1}$, Mohammad Nayeem Hasan ${ }^{2 *}$ and M. A. Taleb ${ }^{2}$ \\ ${ }^{1}$ University of Chittagong, Chittagong, Bangladesh \\ ${ }^{2}$ Shahjalal University of Science \& Technology, Sylhet, Bangladesh
}

*Corresponding author: Mohammad Nayeem Hasan, Shahjalal University of Science \& Technology, Sylhet, Bangladesh. Phone: +8801671912637; E-mail: nayeem5847@gmail.com

Received: 27 February 2021/Accepted: 22 March 2021/ Published: 31 March 2021

\begin{abstract}
Osteoporosis is related to the decrease in bone mineral density. To diagnose osteoporosis and to assess its severity BMD measurement is a widely used method by using dual-energy X-ray absorptiometry (DEXA). BMD is an essential component of the assessment of bone quality and is utilized to assess the osteoporotic status of the bone for the prevention of osteoporotic fractures. The objective of this study was assessed to analyze the correlation between BMD with BMI and age. The study was conducted on 154 patients who performed the BMD test between the periods of January 2018 to July 2019. BMD of the lumbar spine (LS) and right femoral neck (FN) were measured using the DEXA method. In statistical analysis, the BMD status was compared according to age, gender, and BMI. Correlation among BMD, BMI, and age was analyzed with the nonparametric method (spearman rank correlation). SPSS software version 25 was used for analysis. Age showed highly significant negative correlations with all skeletal sites examined. Assessments on the T-score of FN and BMI were significantly related $(\mathrm{p}<0.05, \mathrm{r}=0.223$, positive relation). T-score of LS and BMI were positively correlated $(\mathrm{r}=0.484)$ and it was significant at a $95 \%$ level of significance. Both FN and LS T-score and showed a negative correlation, but it was significant. BMI and age were not significantly associated ( $\mathrm{p}>0.05, \mathrm{r}=-0.080$, negative relation). As expected, the FN T-score and LS T-score showed a high correlation $(r=0.484)$ between each other, and they were positive. Significant correlations were observed among BMD, BMI, and age of the patients. To identify the cause of osteoporosis, BMI and patients age can be considered as risk factors during BMD study. Our hope is that future research will reveal osteoporosis prevention targets effective for the growing population of aging men and women.
\end{abstract}

Keywords: Bone mineral density, dual-energy X-ray absorptiometry, body mass index, fracture

\section{Introduction}

Osteoporosis is a common metabolic bone disorder that leads to increased bone fracture risk. Bone mineral depletion and alteration of bone structure are a common component of aging. The tendency for osteoporosis is steadily increasing as the elderly population grows. Thus, bone strength evaluation has become more important as a diagnostic tool for bone infections (Lee et al., 2019). Osteoporosis was recognized historically when clinical fractures occurred, the development of techniques for bone mass measurement osteoporosis can be identified by studying bone mineral density (BMD). In clinical practice, the diagnosis of osteoporosis is based on the evaluation of bone mass and/ or the presence of fragility fractures (Maghraoui et al., 2009).

World Health Organization (WHO) defined osteoporosis in the year 1994 by an expert panel. World Health Organization densitometric criteria for the diagnosis of osteoporosis when a patient presents with a BMD. Tscore value lower than -2.5 standard deviations were described as being for use in post-menopausal Caucasian women only (Kanis and Kanis, 1994). Thus, it is controversial to use this definition to diagnose osteoporosis in men. For this reason, there is a disparity in the use of WHO criteria in men and there is no consensus about which reference values should be used: those come from bone mass on top of men or who are already acceptable for women (Lin et al., 2013). Since the mid-nineties, it has been well established that it is important 
to achieve as high bone mineral density (BMD) before the late adolescence when reaching the peak of BMD. Calcium and other BMD-associated nutrients are critical for fortifying BMD and for bone health, and it seems prerequisite to increase consumption of calcium-rich and other various food items, and weight-loaded physical exercise from childhood to adolescence (Go et al., 2014; Hasan, 2019; Hasan, 2020). Our lifestyle diet, sleep, defecation, tidiness, dressing etc. are developed and stabilized during childhood, and do not change much later in life. Pre-school dietary preferences are important, but there are few relevant studies.

In this study, we observed the bone mineral density status of suspected patients attended in INMAS, Sylhet. We also analyzed the correlation among BMD and BMI, and verified the relation by relevant literatures.

\section{Methods}

\subsection{Study design}

This is retrospective study including 154 patients (mean \pm SD $57.90 \pm 13.32$ ) who performed BMD test between the periods of January 2018 to July 2019. The age distribution and some other primary parameters are shown in (Table 1). Prior to BMD measurement relevant history (chief complaints, age, sex, height, weight) were taken in all patients. No history of systemic diseases (Hyperparathyroidism, Paget's Disease, Osteocalcin, Renal Osteodystrophy, Osteogenesis Imperfecta), no history of cancer or kidney diseases, no history of hormonal therapy and smoking were taken. The present retrospective study was conducted in the Institute of Nuclear Medicine and Allied Sciences (INMAS), Sylhet. People who underwent DEXA scan from January 2018 to July 2019 formed the study subjects. DEXA scanning was performed by one technician, and the interpretation was done by two nuclear medicine physicians. Height and weight were measured. BMI was calculated from the height and weight recorded while performing the DEXA scan on the formula weight $(\mathrm{kg}) /[\mathrm{height}(\mathrm{m})] 2$. A certified technician measures BMD was done on the femur and lumbar spine (L2 to L4) using a DEXA densitometer. The WHO criteria were used to classify respondents based on DEXA results.

\subsection{Response variable}

In this paper, the dependent variable is Bone Mineral Density (BMD). Here, observation made on the bone mineral density status of suspected patients attended in INMAS, Sylhet and BMD status was compared according to age, gender, and BMI.

The output from the DXA examination includes images of the body part scanned, quantitative data from the scanned area, including the bone mineral content (BMC), BMD, scanned area, T-scores, and Z-scores, and a graph of where the patient fits within the reference population. The results of BMD were expressed as T-scores and we considered that patients with $-2.5 \leq \mathrm{T}$-score $<-1$ had osteopenia and patients with T-score $<-2.5$ had osteoporosis otherwise normal. Besides, this tool used for data collection consisted of sociodemographic details like age, gender and socio-economic condition (Table 1).

\subsection{Predictor variable}

Age of male and female patients (up to 50 years and above 50 years) and Body Mass Index (BMI) (on the basis of underweight, normal and overweight) were used as independent variables.

Table 1. The World Health Organization definitions of osteoporosis and osteopenia used to interpret spine, hip and forearm dual-energy X-ray absorptiometry (DXA) scan results.

\begin{tabular}{ll}
\hline Terminology & T-score definition \\
\hline Normal & $\mathrm{T} \geqslant-1.0$ \\
Osteopenia & $-2.5<\mathrm{T}<-1.0$ \\
Osteoporosis & $\mathrm{T} \leqslant-2.5$ \\
\hline
\end{tabular}

\subsection{Statistical analyses}

Statistics were calculated for continuous variables with mean \pm standard deviation (SD) and categorical variables were expressed as frequencies. Associations between continuous variables were examined by the Spearman correlation coefficient. IBM SPSS 25 was used to performed statistical analyzes; two-tailed $\mathrm{p}<0.05$ was considered statistically significant. 


\section{Results}

The basic anthropometric characteristics of the 154 patients studied are presented in Table 2. The mean age of the patients was $57.90 \pm 13.32 \mathrm{~kg}$ and BMI $24.09 \pm 4.18 \mathrm{~kg} / \mathrm{m}^{2}$. BMD of LS and FN were quantified. The mean \pm SD T-score of FN and LS was $-2.07 \pm 1.72$ and $-1.68 \pm 1.64$, respectively.

Table 2. Characteristics of the study population.

\begin{tabular}{llll}
\hline & Mean (SD) & Minimum & Maximum \\
\hline Age (years) & $57.90(13.32)$ & 23 & 85 \\
BMI $\left(\mathrm{Kg} / \mathrm{m}^{2}\right)$ & $24.09(4.18)$ & 15 & 38 \\
Femur (T-score) & $-2.07(1.72)$ & -4.70 & 9.90 \\
Spine (T-score) & $-1.68(1.64)$ & -5.50 & 3.10 \\
\hline
\end{tabular}

Male and female patients were separately evaluated and divided into two groups according to their age, up to 50 years and above 50 years. In case of FN, it was seen that 54 (35.1\%) cases showed osteopenia, $9(5.8 \%)$ showed osteoporosis and 91 (59.1\%) showed normal BMD. Patients belonging to osteopenia, 46 (85.2\%) were from age group 50+ years and $8(14.8 \%)$ came from less than 50 years of group. According to BMI, $32(59.3 \%)$ were from normal weight and rest of all from overweight or underweight. Among osteoporosis, $45(83.3 \%)$ were female and $9(16.7 \%)$ were male. Patients with osteoporosis, which belongs to two age group showed 8 (88.9\%) from 50+ years and rest from higher age group, 4 (44.4\%) patients showed normal BMI and it was higher from all other BMI group. 7 (77.8\%) were female group.

In Lumber spine, it was seen that $44(28.6 \%)$ cases showed osteopenia, $53(34.4 \%)$ patients has osteoporosis and $57(37.0 \%)$ were from normal BMD. Osteopoenic patients divided into two age groups, $32(72.7 \%)$ were from age group of 50+ years and others $12(27.3 \%)$ were from lower age group. Among osteoporosis, 47 (88.7) were female and rest of all were male.

The osteopenia and osteoporosis according to femur neck, were higher at 50+ years age group and it also similar in lumber spine. According to BMI, osteopenia and osteoporosis was higher in normal weight patients. The age was significant as $\mathrm{p}=0.022$ and $\mathrm{p}=0.031$ from $\mathrm{BMD}$ status for both femur neck and lumber spine, respectively. On the other hand, BMI also significant as $\mathrm{p}=0.044$ and 0.010 from BMD status for both femur and spine. But BMD status was not significant according to patients' gender (Table 3 and 4).

Table 3. BMD status of femur neck in patients.

\begin{tabular}{lllll}
\hline & Normal & Osteopenia & Osteoporosis & P-value \\
\hline $\begin{array}{l}\text { Patients' Age } \\
\leq 50 \text { years }\end{array}$ & $31(34.1)$ & $8(14.8)$ & $1(11.1)$ & 0.022 \\
$\begin{array}{l}50+\text { years } \\
\text { Body Mass Index }\end{array}$ & $60(65.9)$ & $46(85.2)$ & $8(88.9)$ & \\
Underweight & $4(4.4)$ & $6(11.1)$ & $3(33.3)$ & 0.044 \\
Normal & $55(60.4)$ & $32(59.3)$ & $4(44.4)$ & \\
Overweight & $32(35.2)$ & $16(29.6)$ & $2(22.2)$ & \\
Gender & & & & \\
Male & $13(14.3)$ & $9(16.7)$ & $7(22.2)$ & \\
Female & $78(85.7)$ & $45(83.3)$ & $9(5.8)$ & \\
Total & $91(59.1)$ & $54(35.1)$ & $9(5.8)$ & \\
\hline
\end{tabular}


Table 4. BMD status of lumber spine in patients.

\begin{tabular}{lllll}
\hline & Normal & Osteopenia & Osteoporosis & P value \\
\hline $\begin{array}{l}\text { Patients' Age } \\
\text { s50 years }\end{array}$ & $18(31.6)$ & $12(27.3)$ & $10(18.9)$ & \\
50+ years & $39(68.4)$ & $32(72.7)$ & $43(81.1)$ & 0.031 \\
$\begin{array}{l}\text { Body Mass Index } \\
\text { Underweight }\end{array}$ & $2(3.5)$ & $2(4.5)$ & $9(17.0)$ & \\
Normal & $29(50.9)$ & $30(68.2)$ & $32(60.4)$ & 0.010 \\
Overweight & $26(45.6)$ & $12(27.3)$ & $12(22.6)$ & \\
Gender & & & & \\
Male & $14(24.6)$ & $4(9.1)$ & $6(11.3)$ & 0.060 \\
Female & $43(75.4)$ & $40(90.9)$ & $47(88.7)$ & \\
Total & $57(37.0)$ & $44(28.6)$ & $53(34.4)$ & \\
\hline
\end{tabular}

Correlation among BMD, BMI, and age with nonparametric method are listed in Table 5, with spearman rank correlation. Age showed highly significant negative correlations with all skeletal sites examined (Figure 1, Table 5). Assessments of FN BMD and BMI were significantly related ( $\mathrm{p}<0.05, \mathrm{r}=0.223$, positive relation) and LS BMD and BMI were significantly related ( $p<0.05, r=0.484$, positive relation). FN BMD and age were significantly related ( $<<0.05, \mathrm{r}=-0.166$, negative relation) and LS BMD and age were significantly related $(\mathrm{p}<0.05, \mathrm{r}=-257$, negative relation). BMI and age were not significantly associated $(\mathrm{p}>0.05, \mathrm{r}=-0.080$, negative relation). The lumbar spine and femoral neck BMD also showed high correlation between each other ( $<<0.05$, $\mathrm{r}=0.484$, positive relation).

Table 5. Spearman correlation coefficient of bone mineral density (BMD) at different skeletal sites with age and BMI.

\begin{tabular}{lllll}
\hline & Age & BMI & FN BMD & LS BMD \\
\hline Age & 1 & & & \\
BMI & -0.080 & 1 & & \\
FN BMD & $-0.166^{*}$ & $0.223^{* *}$ & 1 & 1 \\
LS BMD & $-0.257^{* *}$ & $0.402^{* *}$ & $0.484^{* *}$ & 1 \\
\hline
\end{tabular}

Correlation types are listed in Fig. 1. Correlation between BMD and age showed linear negative, because dots in graph above were placed in negative axis. As age increases BMD of both sites are decreases and it is below the tolerable score after age $50+$ years.

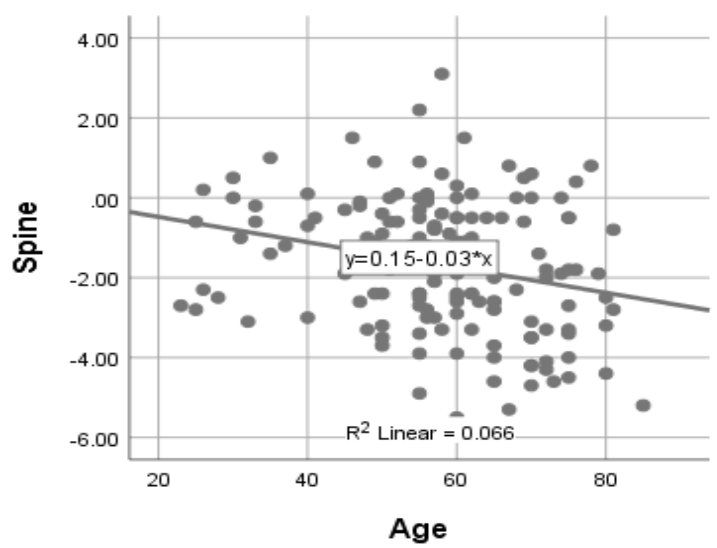

(A)

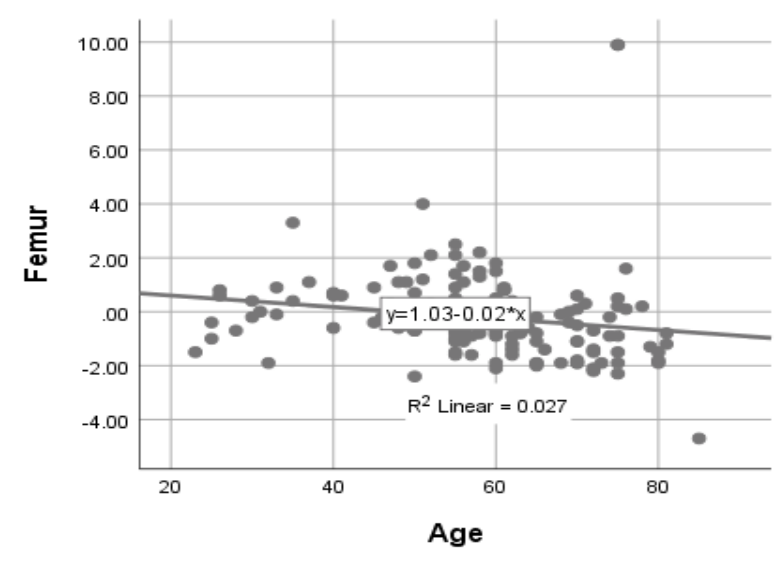

(B)

Figure 1. Scatter-plot of (A) lumbar spine BMD and (B) femur BMD against age.

\section{Discussion}

DEXA artifacts may hinder scan interpretation in obesity because large amounts of soft tissue can increase the apparent density of bone and may obscure bone edges, making the analysis of DEXA data more problematic (Yu et al., 2012). These patterns are even more problematic in determining spinal bone mass, but an overlying 
fat can affect the femur BMD and must be picked up while scanning to ensure proper measurement (Binkley et al., 2003).

However, in this study people with normal BMI are clearly at increased risk low BMD, particularly in spine and femur (Hasan, 2019). A number of studies that have included both sexes and age ranges, confirmed this association (Sharma et al., 2008; Lee et al., 2010). The clarity of the effect of high BMI on BMD incidence is less. An important meta-analysis of seven studies evaluating BMI as a risk factor for BMD showed a nonlinear pattern in the relationship between BMD and BMI (Laet et al., 2005). However, the effect of high BMI on the risk of fracture may vary by physiological site.

The association between obesity and fractures in children has also received recent attention; Goulding and colleagues (Goulding et al., 2000a) first noted that childhood fractures (predominantly forearm) are more frequent in heavier children. In some reports, obese children with fracture had somewhat higher levels of radial bone mineral content (BMC) while in others, fractured kids had lower BMC (Goulding et al., 2000a; Dimitri et al., 2010). One study found that obese men were significantly more at risk for normal weight than men after BMD adjustment. This was especially clear with hip fractures: obese men were five times more likely to have hip fractures than men of normal weight when BMD was considered (Nielson et al., 2011). Why obese men may have a higher risk of fracture than men of normal weight, although obesity reduces the risk of women in some studies, is uncertain (Ferdushi et al., 2020). The thickness of the trochanteric soft tissue is higher in obese women than in thin women, which can reduce the risk of hip fracture by reducing the forces felt by the proximal femur (Bouxsein et al., 2007). One possible explanation for the difference between men and women may be that trochanteric soft tissue thickness in men seemed to be significantly lower than women regardless of BMI (Nielson et al., 2009) and hence the increased fall forces associated with greater weight are not effectively dissipated even in obese men.

This study consistently demonstrated the existence of a significant association between BMD, BMI and BMD, age. The associations of BMI were independent of age or years of the patients. However, as expected, there was an association between FN BMD and LS BMD, Patients with the normal BMI exhibited a 50\% bone loss at the femur and spine. Therefore, by extrapolation, a substantial proportion of these patients are likely to develop osteoporosis before the age of 65 years. That suggest stronger correlation between age and BMD. Furthermore, the correlation between BMI and BMD was acute in spine than femur. These findings suggest a positive effect of body weight on per BMD as compared with percentage with age.

\section{Conclusions}

Osteoporotic fractures occur in the elderly and obese, the importance of these fractures is lost in the message that being overweight increases the risk of fractures. In parallel, a major public health goal is to raise awareness among both patients and health providers of possible osteoporosis and fractures despite having a high BMI. The effectiveness of preventive and treatment strategies should be evaluated in overweight and obese. It is our expectation that future studies will reveal the targets for osteoporosis prevention useful for the aging population of older men and women with high BMI.

\section{Conflict of interest}

None to declare.

\section{References}

Lee YH, JJ Kim, and IG Jang, 2019. Patient-specific phantomless estimation of bone mineral density and its effects on finite element analysis results: a feasibility study. Comput. Math. Methods Med., Volume 2019, Article ID 4102410.

Maghraoui AE, M Ghazi, S Gassim, A Mounach, I Ghozlani, A Nouijai, L Achemlal, A Bezza and M Dehhaoui, 2009. Bone mineral density of the spine and femur in a group of healthy Moroccan men. Bone, 44: 965-969.

Kanis JA and JA Kanis, 1994. Assessment of fracture risk and its application to screening for postmenopausal osteoporosis: Synopsis of a WHO report. Osteoporos. Int., 4: 368-381.

Lin HY, SC Shih, CK Chuang, MR Chen, DM Niu and SP Lin, 2013. Assessment of bone mineral density by dual energy x-ray absorptiometry in patients with mucopolysaccharidoses. Orphanet J. Rare Dis., 8: 71.

Go G, Z Tserendejid, Y Lim, S Jung, Y Min and H Park, 2014. The association of dietary quality and food group intake patterns with bone health status among Korean postmenopausal women: a study using the 2010 Korean National Health and Nutrition Examination Survey Data. Nutr. Res. Pract., 8: 662-669. 
Hasan MN, 2020. Factors associated with attrition of girls students from school in Bangladesh. J. Sci. Res., 12: $29-38$.

Hasan MN, 2019. "A Comparison of Logistic Regression and Linear Discriminant Analysis in Predicting of Female Students Attrition from School in Bangladesh," in 2019 4th International Conference on Electrical Information and Communication Technology, EICT 2019, 2019, doi: 10.1109/EICT48899.2019.9068776.

Yu EW, BJ Thomas, JK Brown and JS Finkelstein, 2012. Simulated increases in body fat and errors in bone mineral density measurements by DXA and QCT. J. Bone Miner. Res., 27: 119-124.

Binkley N, D Krueger and N Vallarta-Ast, 2003. An overlying fat panniculus affects femur bone mass measurement. J. Clin. Densitom., 6: 199-204.

Hasan MN, 2019. Association between self-rated health status and chronic diseases among the elderly in the Sylhet city of Bangladesh. Asian J. Med. Biol. Res., 5: 197-206.

Sharma S, M Fraser, F Lovell, A Reece and AR McLellan, 2008. Characteristics of males over 50 years who present with a fracture: Epidemiology and underlying risk factors. J. Bone Jt. Surg. - Ser. B, 90: 72-77.

Lee SH, YH Khang, KH Lim, BJ Kim, JM Koh, GS Kim, H Kim, NH Cho, 2010. Clinical risk factors for osteoporotic fracture: A population-based prospective cohort study in Korea. J. Bone Miner. Res., 25: 369378.

Laet CD, JA Kanis, A Odén, H Johanson, O Johnell, P Delmas, JA Eisman, H Kroger, S Fujiwara, $\mathrm{P}$ Garnero, EV McCloskey, D Mellstrom, LJ Melton 3rd, PJ Meunier, HAP Pols, J Reeve, A Silman and A Tenenhouse, 2005. Body mass index as a predictor of fracture risk: A meta-analysis. Osteoporos. Int., 16: 1330-1338.

A Goulding, IE Jones, RW Taylor, PJ Manning and SM Williams, 2000a. More broken bones: a 4-year double cohort study of young girls with and without distal forearm fractures. J. Bone Miner. Res., 15: 2011-2018.

Goulding A, RW Taylor, IE Jones, KA McAuley, PJ Manning and SM Williams, 2000b. Overweight and obese children have low bone mass and area for their weight. Int. J. Obes., 24: 627-632.

Dimitri P, JK Wales and N Bishop, 2010. Fat and bone in children: Differential effects of obesity on bone size and mass according to fracture history. J. Bone Miner. Res., 25: 527-536.

Nielson CM, LM Marshall, AL Adams, ES LeBlanc, PM Cawthon, K Ensrud, ML Stefanick, E Barrett-Connor, ES Orwoll and Osteoporotic Fractures in Men Study Research Group, 2011. BMI and fracture risk in older men: the osteoporotic fractures in men study (MrOS). J. Bone Miner. Res., 26: 496-502.

Ferdushi KF, AA Kamil, MN Hasan and T Islam, 2020. "Factors Associated with Coronary Heart Disease among Elderly People in Different Communities," in Statistics for Data Science and Policy Analysis, Springer Singapore, pp. 207-219.

Bouxsein ML, P Szulc, F Munoz, E Thrall, E Sornay-Rendu and PD Delmas, 2007. Contribution of trochanteric soft tissues to fall force estimates, the factor of risk, and prediction of hip fracture risk. J. Bone Miner. Res., 22: $825-831$.

Nielson CM, ML Bouxsein, SS Freitas, KE Ensrud and ES Orwoll, 2009. Trochanteric soft tissue thickness and hip fracture in older men. J. Clin. Endocrinol. Metab., 94: 491-496. 